\title{
Cardiovascular Biofluid Mechanics
}

\author{
Parvati Rajesh \\ Student, Department of Mechanical Engineering \\ Presidency University, Bengaluru, Karnataka, India.
}

\begin{abstract}
This paper intends to study a real-life application of fluid mechanics in cardiovascular blood flow. The study of blood flow is termed as Hemodynamics. Fluid mechanics can be used to analyze the factors and impact of obstruction in blood flow due to fat, cholesterol, and plaque deposits in the coronary arteries of the human heart. These blockages are the grounds for coronary artery diseases and heart attacks. We will look at varying parameters of flowrate and pressure for different thicknesses of epicardial fat as well as define a relationship between these three.
\end{abstract}

Keywords:- Applications of Fluid Mechanics, Biofluid Mechanics, Blood Flow, Cardiology, Cardiovascular Diseases Fluid Mechanics, Heart Attacks, Hemodynamics.

\section{INTRODUCTION}

When we discuss fluids in the body of mammals, the main fluid flow that has our concern is blood flow through the circulatory system. Oxygenated blood flows into the heart through the coronary arteries. When there is an obstruction or blockage in blood flow, this causes deficiency of blood to the heart which may further result in a heart attack if not treated early and cause fatality. This blockage is caused by deposits of fat, cholesterol, and plaque (due to smoking) in the interior lining of the coronary arteries. This deposit is also termed as epicardial fat which can additionally cause local inflammation and possibly have direct consequences on coronary atherosclerosis.

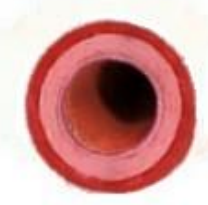

A normal artery

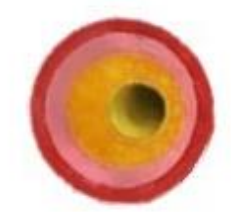
narrows artery
Accumulated Plaque

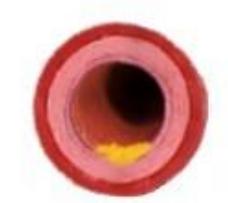

Initial Plaque formation
The thickness of epicardial fat may eventually surge to a magnitude that it entirely impedes the flow of blood and consequently oxygen-rich blood is not delivered to the heart which intern initiates heart failure. The thickness of this fat fluctuates from a minimum of $1 \mathrm{~mm}$ to a maximum measurement of $23 \mathrm{~mm}$.

Biofluid mechanics is progressively further applied in support of diagnosis and decision-making for treatment of clinical pathologies. Exploring the correlation among blood flow phenomena and pathophysiological interpretations is augmented by persistent advances in the imaging modalities, measurement techniques, and abilities of computational models which has greatly enabled us to enhance our understanding.

\section{ANALYSIS}

Arteries can become clogged up due to the existence of plaque especially with contributing factors such as unbalanced diet and no exercise. In Fig. 2, the red lines correspond to the flow of blood around the plague. This depiction is quite like the flow of air around a curved airplane wing section. A low-pressure region has a higher velocity of blood flow whereas the high-pressure region depicts low velocity of blood flow. Due to this pressure difference, there is a possibility of the plaque getting evicted from its initial position and therefore increasing the chances of it blocking an artery with a smaller diameter which can further completely cut off the blood supply to the heart.

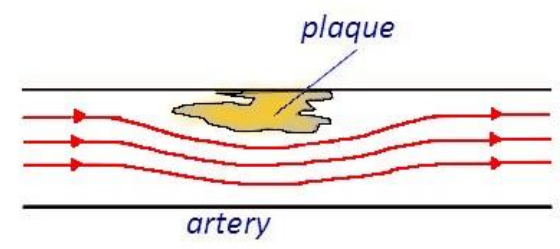

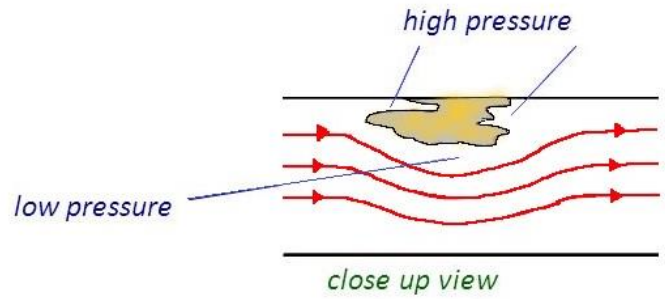

Fig 2:- Plaque Deposition

Fig 1:- Coronary Artery Disease 
The Mean epicardial fat thickness in normal patients and severe coronary syndromes were observed to be $4.4 \pm 1.2 \mathrm{~mm}$ and $6.9 \pm 1.9 \mathrm{~mm}$, respectively. Epicardial fat is linearly associated with the severity of coronary heart disease.

Their thicknesses and volumes can be processed by echocardiography and computed tomography or magnetic resonance imaging. According to a study, the epicardial fat thickness varied between $0.9-13.5 \mathrm{~mm}$ for the 110 individuals that were tested.

Flow rate is defined to be the volume of fluid per unit time flowing past a point through a cross sectional area $A$. Here the shaded cylinder of fluid flows past point $P$ in a pipe of uniform cross-section in time $t$.

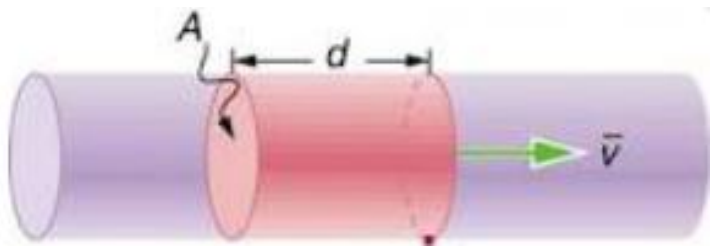

Fig 3:- Nomenclature

All bloods vessels have specific lengths $L$ and inner radii $r$ all through which blood flows. When the pressure in the inlet and outlet are unbalanced, in other words there is a pressure difference $\Delta P$ between the vessel ends, which provides the driving force for flow. Because friction fosters between moving blood and the stationary vessels walls, this fluid movement has a given resistance, which is the measure of how challenging it is to move blood through a vessel. One can then describe a relative relationship between vascular flow, the pressure difference, and resistance also known as the basic flow equation:

$$
\text { Flow }=\frac{\text { Pressure difference }}{\text { Resistances }}
$$

OR

$$
Q=\frac{\Delta P}{R}
$$

where $Q$

$=$ Flow rate (volume/time) $\left(\mathrm{mm}^{3} / \mathrm{sec}\right)$

$\Delta P=$ Pressure difference $(\mathrm{mm}$ of $\mathrm{Hg})$

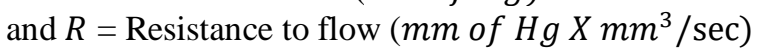

This equation may be relevant not only to a specific vessel but can also be used to define flow through a network. It is known that the resistance to flow through a cylindrical tube or vessel depends on several factors (described by Poiseuille) including:

1) Radius

2) Length

3) Viscosity of the fluid (Blood viscosity)

4) Inherent resistance to flow is as follows:

$$
R=\frac{8 L \eta}{\pi r^{4}}
$$

where $r=$ inside radius of the vessel, $L=$ vessel length and $\eta=$ viscosity of blood.

It is important to note that a small variation in vessel radius will have a very large influence (4th power) on its resistance to flow; e.g., decreasing vessel radius by onehalf will increase its resistance to flow by approximately 16 fold.

If we were to merge the preceding two equations into one expression, which results in the universally known Poiseuille equation, it can be used to better estimate the factors that influence flow through a cylindrical vessel:

$$
Q=\frac{\Delta P \pi r^{4}}{8 L \eta}
$$
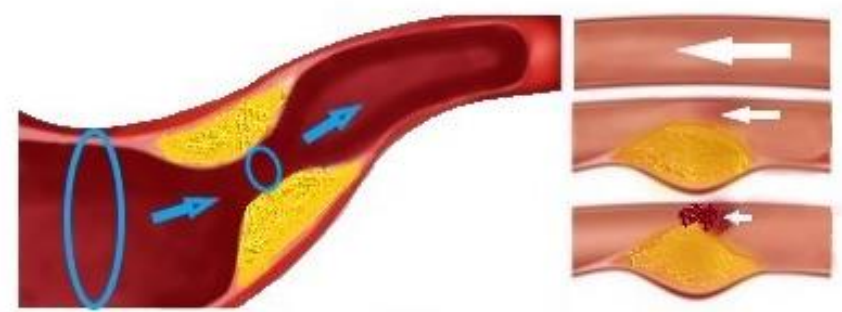

Fig 4:- Flow through a partially blocked artery

\section{COMPUTATION AND GRAPHICAL REPRESENTATION}

The smaller cross-sectional area at the clogging site leads to a faster flow speed due to continuity of flow:

$$
A_{1} V_{1}=A_{2} V_{2}
$$

And causes a drop in pressure, then according to Bernoulli's equation:

$$
P_{2}-P_{1}=\frac{1}{2} \rho\left(V_{2}^{2}-V_{1}^{2}\right)
$$

The pressure drop increases with blood velocity so it is expected to drop more with any physical activity.

Let us now study the pressure and velocity characteristics of blood flowing through the aorta. The aorta is the largest artery in the human body. The ascending aorta branches out to the left and right coronary arteries which further branches out to narrower arteries which are responsible for supplying rich oxygenated blood to the heart. This will be our area of focus now.

The mean diameter of the ascending aorta is $20 \mathrm{~mm}$,

Using continuity equation,

$$
A_{1} V_{1}=A_{2} V_{2}
$$


Mean blood velocity in the ascending aorta

$=11 \mathrm{~cm} / \mathrm{sec}=110 \mathrm{~mm} / \mathrm{sec}$

$$
\begin{gathered}
A_{1}=\pi r^{2} \\
=\pi \times 10^{2} \\
A_{1}=314.159 \mathrm{~mm}^{2}
\end{gathered}
$$

Hence,

$$
A_{1} V_{1}=314.159 \times 110=34557.519 \mathrm{~mm}^{3} / \mathrm{sec}
$$

From equation (1)

$$
\begin{gathered}
V_{2}=\frac{A_{1} V_{1}}{A_{2}} \\
A_{2}=A_{1}-T
\end{gathered}
$$

where $\mathrm{T}=$ epicardial fat thickness $(\mathrm{mm})$

$$
V_{2}=\frac{A_{1} V_{1}}{A_{1}-T}
$$

\begin{tabular}{|c|c|}
\hline $\begin{array}{l}\text { Epicardial fat thickness } \\
(\mathrm{T}), \mathrm{mm}\end{array}$ & $\begin{array}{l}\text { Velocity per unit area } \\
\left(V_{2}\right), \mathrm{mm} / \mathrm{sec}\end{array}$ \\
\hline 3.2 & 111.132 \\
\hline 3.4 & 111.203 \\
\hline 3.6 & 111.275 \\
\hline 3.8 & 111.346 \\
\hline 4.0 & 111.418 \\
\hline 4.2 & 111.490 \\
\hline 4.4 & 111.562 \\
\hline 4.6 & 111.634 \\
\hline 4.8 & 111.706 \\
\hline 5.0 & 111.779 \\
\hline 5.2 & 111.851 \\
\hline 5.4 & 111.923 \\
\hline 5.6 & 111.999 \\
\hline
\end{tabular}

Mean epicardial fat thickness in normal patients ranges from $4.4 \pm 1.2 \mathrm{~mm}$ and the velocities for the above range are calculated using equation (2) and tabulated below:

Table 1
Representing the above values graphically we obtain the following plot:

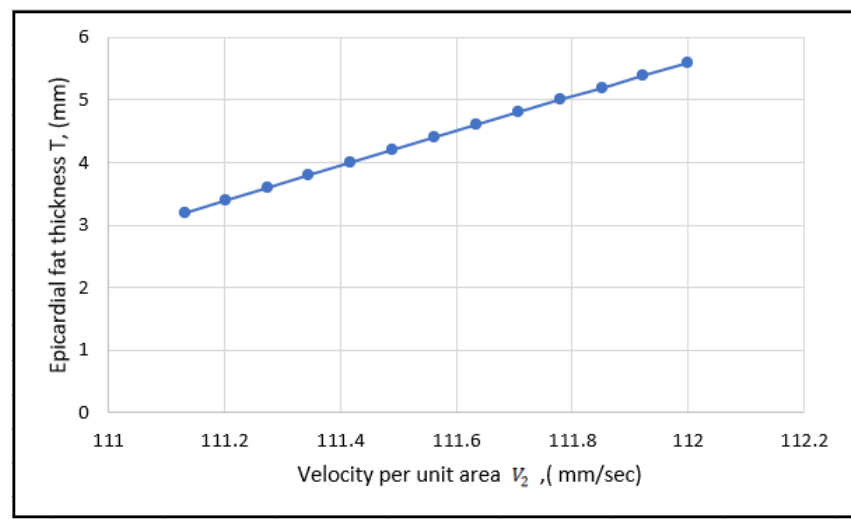

Fig 5

We observe from the plot that as the thickness of epicardial fat increases and lesser the area for blood to flow through freely, the velocity keeps on increasing.

This is similarly observed in the case of patients with severe coronary disorders. The mean epicardial fat in this case is $6.9 \pm 1.9 \mathrm{~mm}$. Similarly, using equation (2), we calculate $V_{2}$ for the range of epicardial fat thicknesses of patients with severe coronary artery diseases.

\begin{tabular}{|c|c|}
\hline $\begin{array}{c}\text { Epicardial fat thickness } \\
(\mathrm{T}), \mathrm{mm}\end{array}$ & $\begin{array}{c}\text { Velocity per unit area } \\
\left(\mathrm{V}_{2}\right), \mathrm{mm} / \mathrm{sec}\end{array}$ \\
\hline 5.9 & 112.105 \\
\hline 6.2 & 112.214 \\
\hline 6.5 & 112.324 \\
\hline 6.8 & 112.433 \\
\hline 7.1 & 112.542 \\
\hline 7.4 & 112.653 \\
\hline 7.7 & 112.763 \\
\hline 8.0 & 112.874 \\
\hline 8.3 & 112.985 \\
\hline 8.6 & 113.096 \\
\hline 8.9 & 113.170 \\
\hline
\end{tabular}

Table 2

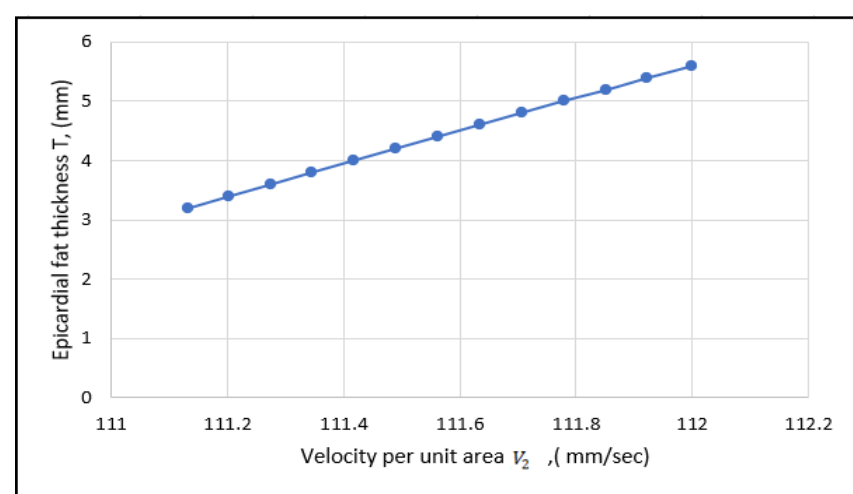

Fig 6

Similar results were obtained which gives us a relation that velocity of blood flow through the aorta is proportional to the epicardial fat thickness. 


\section{PRESSURE RELATION}

To show the relation between epicardial fat thickness and blood pressure through the ascending aorta, we apply the commonly known bernoullis equation.

For this case let us take five random values of epicardial fat thicknesses.

Bernoullis equation;

$\Delta P=\frac{1}{2} \rho\left(V_{2}^{2}-V_{1}^{2}\right)$

$\rho=0.0010565 \mathrm{~g} / \mathrm{mm}^{3}$ (mean density of blood)

Using equation (3)

$\Delta P$ values for the following readings are tabulated below:

\begin{tabular}{|c|c|}
\hline $\begin{array}{c}\text { Epicardial fat thickness } \\
(\mathrm{T}), \mathrm{mm}\end{array}$ & $\begin{array}{c}\text { Pressure difference } \Delta P, \\
\mathrm{~g} / \mathrm{mm}-\mathrm{s}^{2}\end{array}$ \\
\hline 3.2 & 0.132 \\
\hline 4.6 & 0.182 \\
\hline 6.8 & 0.285 \\
\hline 8.0 & 0.338 \\
\hline 8.9 & 0.373 \\
\hline
\end{tabular}

Table 3

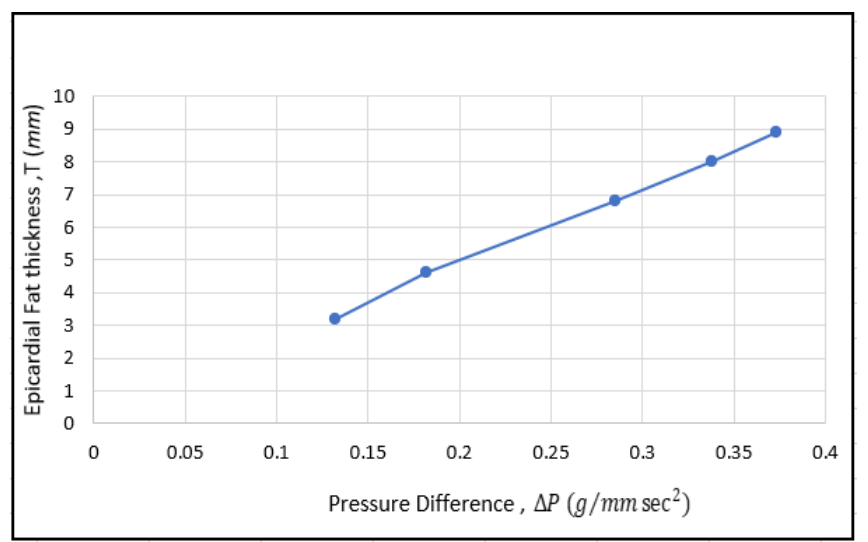

Fig 7

From the graph it can be concluded that blood pressure difference gradually increases with increase in epicardial fat thickness through which we can ascertain our initial claim, and this will lead to a cardiac arrest if not dealt with.

\section{CONCLUSION}

We began from understanding the claims and proceeded to proving it through our various calculationbased readings and interpretation of graphs. We can conclude positively as to how the fats, plaque and cholesterol depositing into the coronary arteries changes the pressure which further increases the velocity( as we observed in plots above) and the reduced area of flow caused by the epicardial fat inevitably leads to a heart problem including heart attacks.
This can be applied to other parts within the body, where these fats or other contaminating particles deposit in the blood vessels and may cause internal clots and other complications. Same can be extended to lymphatic nodes and used to analyze rate of swelling based on the flow rates and pressure differences due to afore mentioned types of blockages. Hence reaching a widespread area in the Biofluid study through fluid mechanics.

\section{REFERENCES}

[1]. Coronary Artery Flow Velocity Is Related To Lumen Area and Regional Left Ventricular Mass- H. Vernon Anderson, Michael J. Stokes, Miltiadis Leon, Subhi A. Abu-Halawa, Yvonne Stuart, and Richard L. Kirkeeide

[2]. Average Coronary Blood Flow Per Unit Weight of Left Ventricle In Patients With And Without Coronary Artery Disease By Francis J. Klocke, M.D., Ivan L. Bunnell, M. D., Davidg Greene, M.D., Stephen M. Wittenberg, M.D., And John P. Visco, M.D.

[3]. Measurement of the Blood Flow Rate and Velocity in Coronary Artery Stenosis Using Intracoronary Frequency Domain Optical Coherence Tomography: Validation Against Fractional Flow Reserve - Haroon Zafar, Faisal Sharif, Martin J Leahy

[4]. Experimental and Computational Methods in Cardiovascular Fluid Mechanics- Charles A. Taylor and Mary T. Draney ST and ALE-VMS

[5]. Methods for patient-specific cardiovascular fluid mechanics modelling -Kenji Takizawa, Yuri Bazilevs, Tayfun E. Tezduyar, Christopher C. Long, Alison L. Marsden and Kathleen Schjodt

[6]. Biofluid Mechanics Richard Skalak And Nihat Ozkaya

[7]. Chandran KB. 2001. Flow dynamics in the human aorta: Techniques and applications. In Cardiovascular Techniques: Biomechanical Systems, Techniques and Applications, ed. CT Leondes, 5:1-25. Boca Raton, FL: CRC Press

[8]. An Introduction to Biofluid Mechanics-Basic Models and Application - Dieter Liepsch 